\title{
Assessment of PhtD C-Terminal Immunogenicity by Opsonophagocytosis Assay (OPA) with OMVs as Adjuvants
}

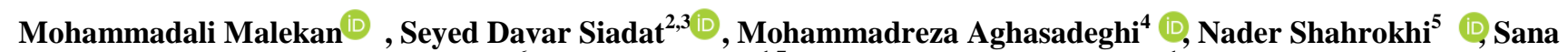 \\ Eybpoosh $^{6}$, Elnaz Afshari ${ }^{1,7} \mathbb{B}$, Seyed Fazlollah Mousavi ${ }^{1 *}$ (1)
}

${ }^{1}$ Department of Microbiology, Pasteur Institute of Iran, Tehran, Iran. ${ }^{2}$ Department of Mycobacteriology\& Pulmonary Research, Pasteur Institute of Iran, Tehran, Iran. ${ }^{3}$ Microbiology Research Center (MRC), Pasteur Institute of Iran, Tehran, Iran. ${ }^{4}$ Viral Vaccine Research Center (VVRC), Pasteur Institute of Iran, Tehran, Iran. ${ }^{5}$ Department of molecular biology, Pasteur Institute of Iran, Tehran, Iran. ${ }^{6}$ Department of Epidemiology and Biostatistics, research centre of emerging infectious diseases, Pasteur Institute of Iran, Tehran, Iran. ${ }^{7}$ Department of Microbiology, Science and Research branch, Islamic Azad university, Tehran, Iran.

\begin{tabular}{|c|c|}
\hline A R T I C L E I N F O & A B S T R A C T \\
\hline $\begin{array}{l}\text { Original Article } \\
\text { VacRes, 2019 } \\
\text { Vol. 6, No. 2, 37-41 } \\
\text { Received: February 29, } 2020 \\
\text { Accepted: June 10, 2020 } \\
\text { Pasteur Institute of Iran } \\
\text { *Corresponding Author: Seyed } \\
\text { Fazlollah Mousavi. Department of } \\
\text { Microbiology, Pasteur Institute of Iran, } \\
\text { Tehran, Iran. } \\
\text { Email: mousavi@pasteur.ac.ir } \\
\text { Tel/Fax: (+98) 9128577041 } \\
\text { KEYWORDS: Streptococcus } \\
\text { pneumonia, Meningococcal OMV, } \\
\text { PhtD, Adjuvant. }\end{array}$ & $\begin{array}{l}\text { Introduction: Streptococcus pneumoniae causes invasive and non-invasive diseases in } \\
\text { children and adults. Currently, there are two types of pneumococcal vaccines: } 23 \text {-valent } \\
\text { pneumococcal polysaccharide vaccine and } 13 \text {-valent pneumococcal conjugate vaccine } \\
\text { which have caused many failures. Therefore, a new generation of pneumococcal vaccines } \\
\text { is being pursued. Methods: An improved version of our previous study was performed } \\
\text { using recombinant C-terminal of pneumococcal polyhistidine triad protein D (PhtD-C) as a } \\
\text { vaccine antigen. The antigen was combined with meningococcal outer membrane vesicle } \\
\text { (OMV) and alum as adjuvants to immunize BALB/c mice intraperitoneally. The generated } \\
\text { total IgG, specific IgG, IgG1 and IgG2a antibodies and the killing ability of pneumococci } \\
\text { by an opsonophagocytosis assay were then assayed. Results: Immunization by } 30 \mu \mathrm{g} \\
\text { PhtD-C and } 50 \mu \mathrm{g} \text { OMV as an adjuvant, induced higher amounts of functional antibodies } \\
\text { compared to our previous study while killing 50-55\% of pneumococci cells. Conclusion: } \\
\text { At optimized concentrations, PhtD-C and meningococcal OMV could be considered as a } \\
\text { potent immunogens and the induced specific IgGs were effective and functional for killing } \\
\text { pneumococci. } \\
\text { Citation: } \\
\text { Malekan M, Siadat S D, Aghasadeghi M, Shahrokhi N, Eybpoosh S, Afshari E et al . Assessment } \\
\text { of PhtD C-Terminal Immunogenicity by Opsonophagocytosis Assay (OPA) with OMVs as } \\
\text { Adjuvants. vacres. 2019; } 6 \text { (2) :37-41. DOI: } 10.29252 / \text { vacres.6.2.37 }\end{array}$ \\
\hline
\end{tabular}

\section{INTRODUCTION}

Streptococcus pneumoniae causes a range of infections in humans, such as pneumonia, otitis media, bacteremia and meningitis [1]. Centers for disease control and prevention (CDC) has reported fatality rates for pneumococcal diseases as follows: pneumonia $(5-7 \%)$, bacteremia ( $20 \%$ in normal people and $60 \%$ in elderly people), and meningitis ( $8 \%$ in children and $22 \%$ in adults) [2]. Annual reports of pneumococcal diseases issued by the World Health Organization and medical centers show that pneumococcal diseases are currently a source of concern in medical settings, and their treatment is accompanied by major troubles emerging by antimicrobial resistance between pneumococcal isolates [3]. Evidence suggests that the antimicrobial resistance of pneumococcal strains is increasing around the world. In the treatment of pneumococcal infections, resistance to beta-lactams, macrolides, lincosamides, fluoroquinolones, tetracyclines and trimethoprimsulfamethoxazole is considerable [2]. Two types of vaccines for protection against pneumococcal diseases, including 23-valent pneumococcal polysaccharide vaccine (PPV) and pneumococcal conjugate vaccine (PCV) (PCV-7, PCV-10, and PCV-13), are currently used by different countries. The introduction of these vaccines provided a useful strategy for the prevention of pneumococcal infections and decreased their prevalence [4]. However, significant limitations have also emerged with the use of these vaccines, such as the high cost of $\mathrm{PCV}$, serotype-dependency of the vaccines, replacement of non-vaccine serotypes, and lack of effectiveness of PPV in children less than 2 years old. Therefore, efforts have been made to resolve these issues and invent new strategies to develop a new generation of pneumococcal vaccines that are not dependent on pneumococcal serotypes. The most significant types of such new vaccines are based on pneumococcal proteins [5]. Currently, a group of pneumococcal proteins are candidates for the prevention of pneumococcal diseases [6]. The polyhistidine triad (Pht) protein family is considered as a major candidate among such proteins. The Pht protein family consists of four members: PhtA, PhtB, PhtD and PhtE. All the members are pneumococcal surface proteins containing 5 to 6 histidine 
triad motifs $(\mathrm{HxxHxH})$. PhtD has been considered as a protein with full coverage between all pneumococcal strains; therefore, it has been selected as an important agent in new protein-based pneumococcal vaccines [7]. Four types of Pht proteins (A, B, $\mathrm{D}$, and $\mathrm{E}$ ) have been shown as protective proteins. Among them, PhtD is the most protective immunogen in animal models of pneumococcal diseases [8-10]. Different adjuvants have been experienced with $\mathrm{PhtD}$, and different immunization routes have been applied for PhtD immunogenicity assessments [11]. Some studies have indicated that the C-terminal of PhtD is more surface-exposed than the other regions of PhtD which could make it a potential immunogenic fragment [7, 12, 13]. The combination of PhtD with alum has been examined in phase I clinical trials. Therefore, PhtD can be a promising vaccine candidate [14].

Although alum as an adjuvant has been used in combination with $\mathrm{PhtD}$, a limited number of biological agents have been used as adjuvants in vaccine preparations. The heatlabile enterotoxin of Escherichia coli (LT) and outer membrane vesicles (OMVs) of Gram-negative bacteria as adjuvants of PhtD have been applied in some studies [11]. Budding round structures, protruding from the outer membrane of Gramnegative bacteria consist of proteins and lipids, especially lipopolysaccharide (LPS) and periplasmic contents. Several functions were found for OMVs, like the formation of biofilms and transportation of virulence factors to new environments, such as host cells.

Presently, there are several types of adjuvants that are used in many types of vaccines. Some of these adjuvants have proven as effective while some others have not been accepted as routine adjuvants and more studies are needed to prove their effectiveness. Bacterial OMVs are among these adjuvants. A few types of bacterial OMVs have been studied so far, among which meningococcal OMV is the most prevalent one. Meningococcal OMV has been studied in terms of toxicity, side effects and functions, and has shown to be harmless without any adverse effects as an adjuvant. The conformational structure and material components of meningococcal OMV are highly critical in antigen delivery to the immune system cells $[15,16]$. Naturally, S. pneumoniae can colonize the upper respiratory tract of humans and can cause different infections in people with disabilities and underlying diseases as well as children and the elderly. As previously mentioned, pneumococci can cause a range of infections, including mild and severe diseases. Pneumonia, otitis media, and sinusitis are respiratory diseases; however, severe and invasive infections such as sepsis and meningitis are occurred by blood or the systemic routes [1].

We had previously assayed a combination of PhtD and PhtD-C with alum and meningococcal OMV which exhibited modest results in generating antibodies and increasing survival rates of the immunized mice[17]. In this study, we only used the C-terminal of PhtD which is considered as the first-line epitope that would inhibit the binding of complement proteins on the pneumococcal surface [10]. Moreover, we assayed the generated antibodies in immunized $\mathrm{BALB} / \mathrm{c}$ mice with increased concentrations of the anigen and the menagococcal OMV and performed an opsonophagocytosis assay which all enhanced our previous results.

\section{MATERIALS AND METHODS}

\section{Animals and the Ethics Statement}

Female BALB/c mice (6-8 weeks) were provided by the Pasteur Institute of Iran under standard protection and ethical supervision. The Ethics Committee of Pasteur Institute of Iran approved all procedures herein performed.

\section{Adjuvants}

Alum, as an adjuvant, was commercially available (\#77161 Thermo Fisher Scientific, USA) as an already prepared liquid and was used as recommended by the manufacturer. Neisseria meningitidis serogroup A strain (G243/CSBPI) OMV as a bioadjuvant was prepared, purified and confirmed for safety, as described previously[17].

\section{Immunizations}

Four groups of antigen combinations and 3 groups of negative controls were prepared for the immunizations as described in Table 1. Each group was consisted of 10 mice. The injectable material were incubated at room temperature for 30 min. The mice were intraperitoneally immunized on days 0,14 , and 28 .

Table 1. The intraperitoneally immunized BALB/c mice groups.

\begin{tabular}{|c|c|c|c|}
\hline $\begin{array}{c}\text { Group } \\
N^{o} .\end{array}$ & $\begin{array}{l}\text { Immunized } \\
\text { with }\end{array}$ & concentration and volumes & $\begin{array}{c}\text { Total volume } \\
\text { injected } \\
\text { per mouse }\end{array}$ \\
\hline 1 & $\begin{array}{l}\text { PhtD-C } \\
+\mathrm{OMV}\end{array}$ & $\begin{array}{c}\text { [PhtD-C]: } 0.6 \mu \mathrm{g} / \mu \mathrm{l} \text { in PBS } \\
\text { PhtD-C volume: } 50 \mu 1 \\
\text { [OMV]: } 1 \mu \mathrm{g} / \mu \mathrm{l} \text { in PBS } \\
\text { OMV volume: } 50 \mu \mathrm{l}\end{array}$ & $100 \mu 1$ \\
\hline 2 & $\begin{array}{l}\text { PhtD-C } \\
\text { +alum }\end{array}$ & $\begin{array}{c}\text { PhtD-C]: } 0.6 \mu \mathrm{g} / \mu \mathrm{l} \text { in PBS } \\
\text { PhtD-C volume: } 50 \mu \mathrm{l} \\
\text { Alum volume: } 50 \mu \mathrm{l}\end{array}$ & $100 \mu 1$ \\
\hline 3 & PhtD-C & $\begin{array}{c}\text { [PhtD-C]: } 0.6 \mu \mathrm{g} / \mu 1 \text { in PBS } \\
\text { PhtD-C volume: } 100 \mu \mathrm{l}\end{array}$ & $100 \mu 1$ \\
\hline 4 & $\begin{array}{c}\text { PBS + } \\
\text { alum } \\
\text { (negative } \\
\text { control) }\end{array}$ & $\begin{array}{l}\text { PBS volume: } 50 \mu 1 \\
\text { Alum volume: } 50 \mu 1\end{array}$ & $100 \mu 1$ \\
\hline 5 & $\begin{array}{c}\text { PBS + } \\
\text { OMV } \\
\text { (negative } \\
\text { control) }\end{array}$ & $\begin{array}{c}\text { PBS volume: } 50 \mu \mathrm{l} \\
{[\mathrm{OMV}]: 1 \mu \mathrm{g} / \mu \mathrm{l} \text { in PBS }} \\
\text { OMV volume : } 50 \mu \mathrm{l}\end{array}$ & $100 \mu 1$ \\
\hline 6 & $\begin{array}{c}\text { PBS } \\
\text { (negative } \\
\text { control) }\end{array}$ & $100 \mu \mathrm{l}$ & $100 \mu 1$ \\
\hline
\end{tabular}

The blood sampling of the immunized mice and the negative controls was performed from the posterior vena cava on days 14,28 , and 35 . The blood samples were collected from each mouse per group $(n=10)$ and let to coagulate at room temperature for $30 \mathrm{~min}$, and then, serum was separated with centrifugation and stored at $-20^{\circ} \mathrm{C}[10]$.

\section{Evaluation of Antibodies}

Levels of total IgG, specific IgG, IgG1, and IgG2a in the serum samples were evaluated in the immunized mice using two types of ELISA. The first type was performed for the assessment of specific IgG, IgG1, and IgG2a, while the second type was performed for total IgG evaluation. Total IgG was evaluated using IgG (Total) Mouse Uncoated ELISA Kit (cat. \# 
88-50400; Invitrogen, USA). Specific IgG and its two subclasses (IgG1 and IgG2a) were evaluated by ELISA method. Three $\mu \mathrm{g}$ of PhtD-C in $100 \mu \mathrm{l}$ of PBS were coated in a Nunc Maxisorp 96-well plate and incubated overnight $\left(4^{\circ} \mathrm{C}\right)$. The evaluation of specific IgG, IgG1, and IgG2a using ELISA method was similar. However, the HRP-conjugated secondary antibody was different for specific $\operatorname{IgG}, \operatorname{IgG} 1$, and $\operatorname{IgG} 2$ a as follows: 1- Goat anti-Mouse IgG cat. \# 62-6520; 2- Goat antiMouse IgG1 cat. \# PA1-74421; 3- Goat anti-Mouse IgG2a cat. \# A-10685; respectively; all by Thermo Fisher Scientific, USA.

\section{Opsonophagocytosis Assay (OPA)}

OPA is a test for the assessment of functional antibodies against pneumococcal vaccines and proteins. Phagocytosis and killing of pneumococci by leukocytes via stimulating and inducing functional antibodies form the basis of this assay. Goyette-Desjardins and Melin described this test for some invasive encapsulated extracellular bacteria, such as Streptococcus pneumonia $[18,19]$. We used the procedure as described previously[17].

\section{Statistical analysis}

The results related to the ELISA assays and OPA were analyzed using SPSS software. Antibody levels and OPA results related to each antigen group on days 14, 28, and 35 are described in the column diagram. The change of antibody levels and percentage of the killed bacteria in the OPA test were indicated in each time section and tested using Friedman nonparametric test. Kruskal-Wallis test was also applied for comparison of the results on day 35 .

\section{RESULTS}

\section{Serum Levels of Antibodies}

The total $\mathrm{IgG}$ level, as well as specific $\mathrm{IgG}, \mathrm{IgG} 1$, and IgG2a in serum samples of the immunized mice from days 14 , 28 , and 35 indicated that there was an ascending pattern in antibody levels from day 14 to 35 for total $\mathrm{IgG}$, specific $\mathrm{IgG}$, and $\operatorname{IgG} 1$, but not for $\operatorname{IgG} 2 \mathrm{a}$. It could be inferred that booster doses of antigens could rise the total $\mathrm{IgG}$, specific $\mathrm{IgG}$, and IgG1 antibody production. This rising pattern was not observed in IgG2a. Fig. 1 indicates the total IgG levels on day 35. This figure shows significant levels of $\mathrm{IgG}$ total against PhtD$\mathrm{C}+$ alum and PhtD-C+OMV. The negative controls (i.e. PBS, $\mathrm{PBS}=$ alum and PBS+OMV), showed a basal amount of total IgG levels because the mice were already exposed to foreign antigens.

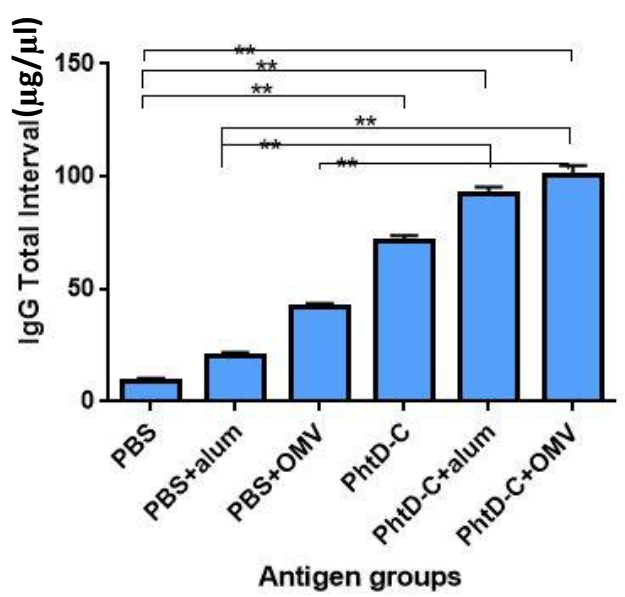

Fig.1. Total IgG levels on day 35 indicated significant levels of total $\mathrm{IgG}$ for two groups of PhtD-C+alum and PhtD-C+OMV. No significant difference was observed among the antigen groups $(P$-value $=0.2)$, but a significant difference was detected between the negative controls and the antigen groups $(P$-value $<0.001)$.
Levels of specific IgG against the antigen combination are indicated in Fig. 2. It could be inferred from this figure that specific IgG had a significant level against PhtD-C+alum and PhtD-C+OMV. Specific IgG was not detected for the negative controls.

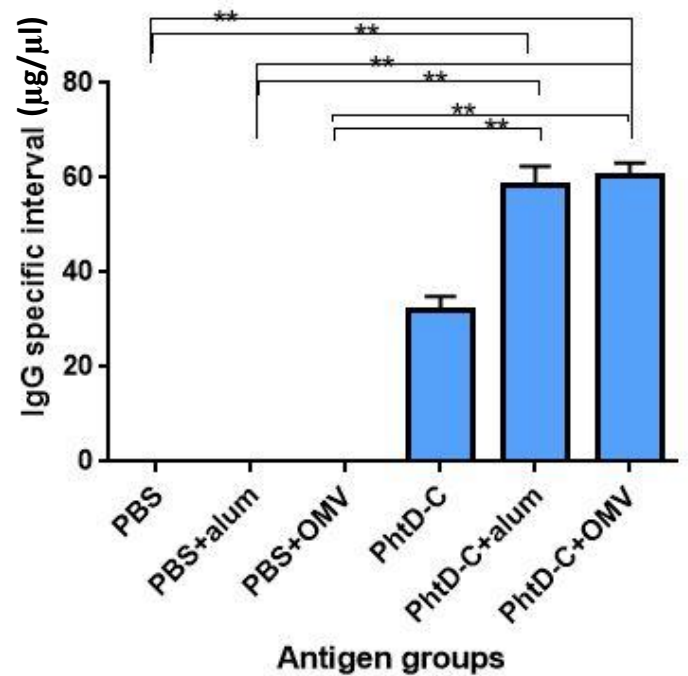

Fig. 2. The amount of IgG specific was equally similar against PhtD$\mathrm{C}+$ alum and PhtD-C+OMV, and they were comparable with total IgG results. The negative controls showed no result. No significant difference was observed among the antigen groups ( $P$-value 0.2$)$, but a significant difference was detected between the negative controls and the antigen groups $(P$-value $<0.001)$.

Two subclasses of specific $\operatorname{IgG}$ (i.e. $\operatorname{IgG} 1$ and $\operatorname{IgG} 2 \mathrm{a}$ ) were evaluated in this study.

No rising pattern was detected in IgG2a from day 14 to 35 . It could be inferred that the immune system was not stimulated for IgG2a production. The negative controls showed no response.

As mentioned in the materials and methods section, the ELISA method for assessment of IgG1 and IgG2a was similar with specific $\mathrm{IgG}$, but differed in HRP-conjugated secondary IgG. As indicated in Fig. 3, $80 \%$ of IgG specific against the antigens in this study accounted for IgG1. Levels of IgG1 against PhtD-C+alum and PhtD-C+OMV were similar to those of specific $\mathrm{IgG}$ against such combinations. The negative controls showed no result.

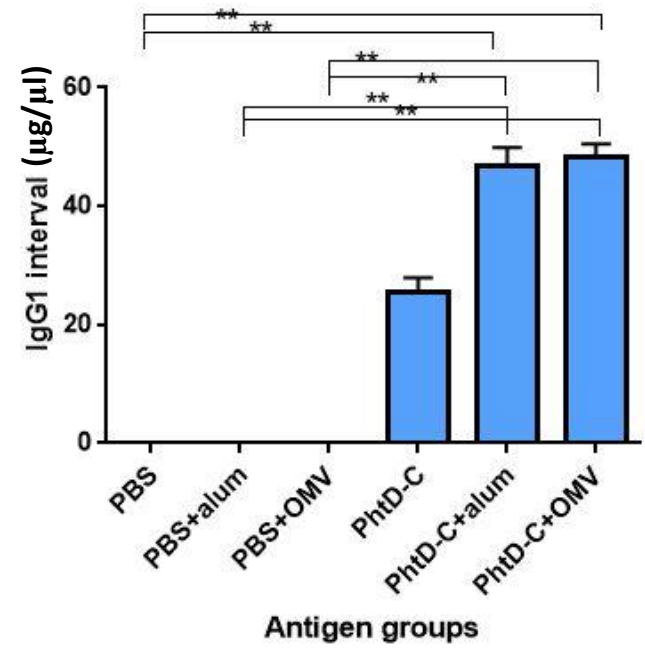

Fig.3. Amounts of PhtD-C+alum and PhtD-C+OMV IgG1 levels were comparable with levels of specific IgG. No significant difference was shown among the antigen groups ( $P$-value 0.2$)$, but a significant difference was detected between the negative controls and the antigen groups $(P$-value $<0.001)$. 


\section{OPA}

Since the level of antibodies could not reveal their functions for triggering the immune responses; it was necessary to evaluate the functional antibodies by OPA. As depicted in Fig. 4, OPA indicated that a large number of pneumococci (i.e. $55 \%$ ) were killed by functional antibodies in the serum samples of mice immunized with PhtD-C+alum in third period of the immunization. A comparison between this combination and PhtD-C+OMV showed that the combination with OMV as a bio-adjuvant was comparable $(50 \%)$ with the combination with a chemical adjuvant such as alum. Meanwhile, no effect was detected for the negative controls.

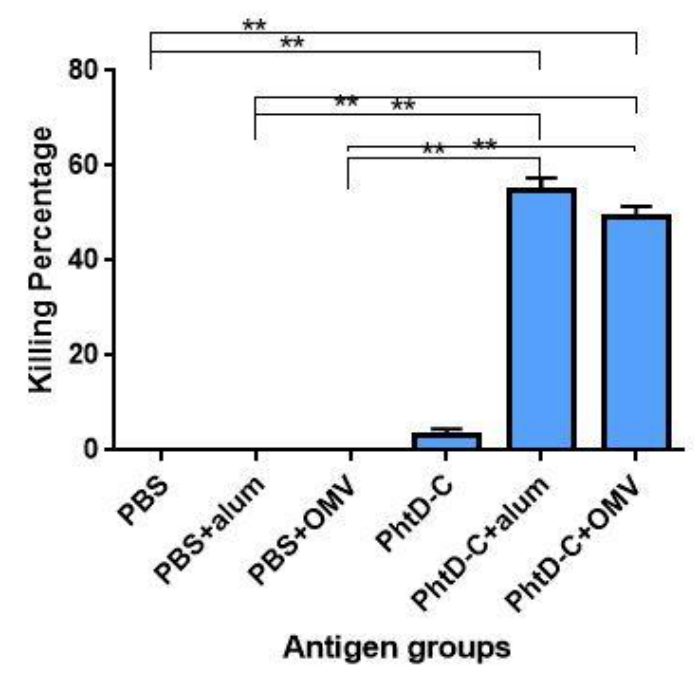

Fig.4. OPA indicated that combinations of PhtD-C+alum and PhtD$\mathrm{C}+\mathrm{OMV}$ inducing phagocytosis and killing pneumococci in the presence of functional specific IgG. A considerable difference was observed between PhtD-C+alum, PhtD-C+OMV, and PhtD-C $(P$-value $<0.001)$.

\section{DISCUSSION}

S. pneumoniae as a normal colonizer in upper respiratory tracts of humans can potentially be an opportunist pathogen and cause a range of diseases like pneumonia, sinusitis, otitis media, sepsis, and meningitis [1]. Pneumococci are typed based on their capsular polysaccharides, and 98 types of these polysaccharides have been detected so far [4]. Approximately 40 virulence factors of pneumococci can create a wide range of diseases in humans. The most important virulence factor of pneumococci are their polysaccharide capsule [20]. Many of the patient's complaints in their referral to health and medical centers are about pneumococcal infections. Over time, pneumococci have naturally selected modification systems for their capabilities in changing capsular polysaccharides.

Due to these modifications, several serotypes have emerged around the world, and vaccine serotype replacement has been prompted this phenomenon. Serotype replacement with non-vaccine serotypes, different geographical patterns of serotypes, and effectiveness failure in the elderly or disabled people are among the main shortcomings of the current vaccines. Thus, scientists have made efforts to develop new vaccines that are not dependent on the serotypes. Some pneumococcal proteins have been selected as candidates for these groups of vaccine [21-23]. A group of surface pneumococcal proteins, known as poly-histidine triad (Pht) proteins, is recognized as proper candidates for the development of new pneumococcal vaccines [4, 24, 25]. Four proteins (i.e. PhtA, PhtB, PhtD, and PhtE) have been identified based on their immunogenicity features and have been evaluated. Accordingly, some studies have examined the potential of $\mathrm{PhtD}$, its derivatives, routes of immunizations and combinations with different adjuvants as immunogens [10, 21, $26,27]$. There are two studies which have used OMV as an adjuvant. Kuipers and colleagues from 2015 to 2017 conducted two separate studies, in which Salmonella OMV was used as an adjuvant for PspA and Ply pneumococcal proteins in pulmonary immunization [28]. The properties of meningococcal OMV from serogroup A were previously studied by Siadat and colleagues [29]. Different routes of immunization with pneumococcal antigens were investigated in immunization studies. Among them, respiratory, intramuscular and intraperitoneal routes are more common than others. It should be mentioned that respiratory or nasal route can be specified for the confrontation of mucosal and innate immunity with pneumococcal antigens, and no specific immune system is probably involved with these antigens.

One of the earliest and pioneering studies on Pht proteins and PhtD was conducted by Adamou and colleagues (2001). They focused on the immunogenicity of the PhtD+Freund adjuvant in $\mathrm{C} 3 \mathrm{H} / \mathrm{Hej}$ mice using the intradermal route [8]. Godfroid and colleagues (2011) used Pht proteins in their preclinical study and administered them with different routes of immunization and concluded that antibody induction was not reliable in respiratory and intramuscular routes [11]. Danoe and colleagues (2011) immunized macaque rhesus with PhtD using the intramuscular route. They combined this protein with ASO2 as the adjuvant and concluded that the functions of antibodies were not reliable [21]. One of the most similar studies to our study was performed by Plumptre and colleagues (2013) in which they used truncated fragments of PhtD, although they did not exactly define the $\mathrm{C}$-terminal or other parts of this protein and chose the intramuscular route of immunization [10]. Verhoven and coworkers (2014) investigated the immunization of mice cubs using the intramuscular route of PlyD1+PcpA+PhtD and alum as the adjuvant. They reported that antibodies produced after this immunization were functional against pneumonic bacteremia [30].

The current study was a modified and improved version of our previous study[17] in which we used both total and Cterminal of $\mathrm{PhtD}$ combined with menangococal OMV and alum, albeit at lower concentrations[17]. Based on previous studies on PhtD as an immunogen, we assessed its C-terminal since it protrudes from the outer surface of pneumococcal cells and it could be exposed to the immune system to induce production of functional antibodies. Compared to our previous study in which we had immunized each BALB/c mouse with 10 $\mu \mathrm{g}$ PhtD-C and $10 \mu \mathrm{g}$ OMV, here we used $30 \mu \mathrm{g}$ PhtD-C and $50 \mu \mathrm{g}$ OMV which resulted in significant differences in productions of total $\mathrm{IgG}$, specific $\mathrm{IgG}$ and $\mathrm{IgG} 1$ with functional antibodies capable of more than $50 \%$ killing of pneumococci cells. Interestingly, the above-mentioned amount of the OMV was as efficient as the recommended amounts of alum per injection. The levels of total $\mathrm{IgG}$ were naturally higher than those of specific IgG and IgG1 because the mice faced other environmental antigens previously and before the immunization. The results of total $\mathrm{IgG}$ in the negative controls showed a partially elevated pattern. The results of the negative controls also confirmed the total IgG results. As mentioned earlier, a rising pattern was observed in the production of total $\mathrm{IgG}$, specific $\mathrm{IgG}$, and $\mathrm{IgG} 1$ from the first to third 
immunization. This pattern indicated that the process of antibody production was amplified after days 14 and 28; however, there was no such rising pattern in $\mathrm{IgG} 2 \mathrm{a}$.

These results suggested that the dominant immune responses were the humoral type involving helper T2 lymphocytes. Moreover, PhtD-C+alum and PhtD-C+OMV presented significant levels of antibodies. The optimization of the OMV and PhtD-C concentrations as well as more detailed evaluations of the toxicity of this formulation are required to be assessed by the future studies.

\section{ACKNOWLEDGEMENTS}

The authors thank the personnel of microbiology department, Department of Mycobacteriology \& Pulmonary Research and Microbiology Research Center (MRC) in Pasteur Institute of Iran for their assistance and cooperation in this project.

\section{CONFLICTS OF INTEREST}

The authors declare that they have no known competing financial interests or personal relationships that could have appeared to influence the work reported in this paper.

\section{REFERENCES}

1. Weiser JN, Ferreira DM, Paton JC. Streptococcus pneumoniae: transmission, colonization and invasion. Nature Reviews Microbiology. 2018;16(6):355-67. doi:10.1038/s41579-018-0001-8.

2. Book CP. Immunology and vaccine-preventable diseases, Pink Book of CDC - Pneumococcal diseases. 2019. p. $279-96$.

3. Control CfD, Prevention. Effects of new penicillin susceptibility breakpoints for Streptococcus pneumoniae--United States, 2006-2007. MMWR Morbidity and mortality weekly report. 2008;57(50):1353-57.

4. Feldman C, Anderson R. Review: Current and new generation pneumococcal vaccines. The Journal of infection. 2014;69(4):309-25. doi:10.1016/j.jinf.2014.06.006.

5. Darrieux M, Goulart C, Briles D, Leite LCdC. Current status and perspectives on protein-based pneumococcal vaccines. Crit Rev Microbiol. 2015;41(2):190-200. doi:10.3109/1040841x.2013.813902.

6. Lagousi T, Basdeki P, Routsias J, Spoulou V. Novel protein-based pneumococcal vaccines: assessing the use of distinct protein fragments instead of full-length proteins as vaccine antigens. Vaccines (Basel). 2019;7(1):9. doi:10.3390/vaccines7010009.

7. Rioux S, Neyt C, Di Paolo E, Turpin L, Charland N, Labbe S et al. Transcriptional regulation, occurrence and putative role of the Pht family of Streptococcus pneumoniae. Microbiology (Reading, England). 2011;157(Pt2):336-48. doi:10.1099/mic.0.042184-0.

8. Adamou JE, Heinrichs JH, Erwin AL, Walsh W, Gayle T, Dormitzer M et al. Identification and characterization of a novel family of pneumococcal proteins that are protective against sepsis. Infection and immunity. 2001;69(2):949-58. doi:10.1128/iai.69.2.949-958.2001.

9. Hamel J, Charland N, Pineau I, Ouellet C, Rioux S, Martin D et al. Prevention of pneumococcal disease in mice immunized with conserved surface-accessible proteins. Infection and immunity. 2004;72(5):2659-70. doi:10.1128/iai.72.5.2659-2670.2004.

10. Plumptre $\mathrm{CD}$, Ogunniyi $\mathrm{AD}$, Paton JC. Vaccination against Streptococcus pneumoniae using truncated derivatives of polyhistidine triad protein D. PloS one. 2013;8(10):e78916. doi:10.1371/journal.pone.0078916 11. Godfroid F, Hermand P, Verlant V, Denoël P, Poolman JT. Preclinical Evaluation of the Pht Proteins as Potential Cross-Protective Pneumococcal Vaccine Antigens. Infection and immunity. 2011;79(1):238. doi:10.1128/IAI.00378-10.

12. Beghetto E, Gargano N, Ricci S, Garufi G, Peppoloni S, Montagnani F et al. Discovery of novel Streptococcus pneumoniae antigens by screening a whole-genome $\lambda$-display library. FEMS microbiology letters. 2006;262(1):14-21. doi:10.1111/j.1574-6968.2006.00360.x.

13. Malley R, Anderson PW. Serotype-independent pneumococcal experimental vaccines that induce cellular as well as humoral immunity.
Proc Natl Acad Sci U S A. 2012;109(10):3623-7. doi:10.1073/pnas.1121383109.

14. Seiberling M, Bologa M, Brookes R, Ochs M, Go K, Neveu D et al. Safety and immunogenicity of a pneumococcal histidine triad protein D vaccine candidate in adults. Vaccine. 2012;30(52):7455-60. doi: $10.1016 /$ j.vaccine. 2012.10 .080 .

15. Jan AT. Outer membrane vesicles (OMVs) of gram-negative bacteria: a perspective update. Frontiers in microbiology. 2017;8(1053). doi:10.3389/fmicb.2017.01053.

16. Moshiri A, Dashtbani-Roozbehani A, Najar Peerayeh S, Davar Siadat S. Outer membrane vesicle: a macromolecule with multifunctional activity. Human vaccines \& immunotherapeutics. 2012;8(7):953-5. doi: $10.4161 / \mathrm{hv} .20166$

17. Malekan M, Siadat SD, Aghasadeghi M, Shahrokhi N, Afrough P, Behrouzi A et al. Evaluation of protective immunity responses against pneumococcal PhtD and its C-terminal in combination with outermembrane vesicles as adjuvants. Journal of Medical Microbiology. 2020;69(3):465-77. doi:10.1099/jmm.0.001103.

18. Goyette-Desjardins G, Roy R, Segura M. Murine whole-blood opsonophagocytosis assay to evaluate protection by antibodies raised against encapsulated extracellular bacteria. In: Lepenies B, editor. Carbohydrate-Based Vaccines: Methods and Protocols. New York, NY: Springer; 2015. p. 81-92.

19. Melin M, Di Paolo E, Tikkanen L, Jarva H, Neyt C, Käyhty H et al. Interaction of pneumococcal histidine triad proteins with human complement. Infection and immunity. 2010;78(5):2089-98. doi:10.1128/iai.00811-09.

20. Barendt SM, Sham L-T, Winkler ME. Characterization of mutants deficient in the L, D-carboxypeptidase (DacB) and WalRK (VicRK) regulon, involved in peptidoglycan maturation of Streptococcus pneumoniae serotype 2 strain D39. Journal of bacteriology. 2011;193(9):2290-300. doi:10.1128/jb.01555-10.

21. Denoël P, Philipp MT, Doyle L, Martin D, Carletti G, Poolman JT. A protein-based pneumococcal vaccine protects rhesus macaques from pneumonia after experimental infection with Streptococcus pneumoniae. Vaccine. 2011;29(33):5495-501. doi:10.1016/j.vaccine.2011.05.051.

22. Ogunniyi AD, Mahdi LK, Trappetti C, Verhoeven N, Mermans D, Van der Hoek MB et al. Identification of genes that contribute to the pathogenesis of invasive pneumococcal disease by in vivo transcriptomic analysis. Infection and immunity. 2012;80(9):3268-78. doi:10.1128/iai.00295-12.

23. Giefing C, Meinke AL, Hanner M, Henics T, Minh DB, Gelbmann D et al. Discovery of a novel class of highly conserved vaccine antigens using genomic scale antigenic fingerprinting of pneumococcus with human antibodies. The Journal of experimental medicine. 2007;205(1):117-31. doi:10.1084/jem.20071168.

24. Ogunniyi AD, Grabowicz M, Briles DE, Cook J, Paton JC. Development of a vaccine against invasive pneumococcal disease based on combinations of virulence proteins of Streptococcus pneumoniae. Infection and immunity. 2007;75(1):350-7. doi:10.1128/iai.01103-06.

25. Hicks LA, Harrison LH, Flannery B, Hadler JL, Schaffner W, Craig AS et al. Incidence of pneumococcal disease due to non-pneumococcal conjugate vaccine (PCV7) serotypes in the United States during the era of widespread PCV7 vaccination, 1998-2004. The Journal of infectious diseases. 2007;196(9):1346-54. doi:10.1086/521626.

26. Plumptre CD, Ogunniyi AD, Paton JC. Polyhistidine triad proteins of pathogenic streptococci. Trends in microbiology. 2012;20(10):485-93. doi:10.1016/j.tim.2012.06.004.

27. Khan M, Pichichero M. CD4 T cell memory and antibody responses directed against the pneumococcal histidine triad proteins $\mathrm{PhtD}$ and $\mathrm{PhtE}$ following nasopharyngeal colonization and immunization and their role in protection against pneumococcal colonization in mice. Infection and immunity. 2013;81(10):3781-92. doi:10.1128/iai.00313-13.

28. Kuipers K, Jong WS, van der Gaast-de CE, Houben D, van Opzeeland F, Simonetti E et al. Th17-mediated cross protection against pneumococcal carriage by vaccination with a variable antigen. Infection and immunity. 2017;85(10):e00281-17. doi:10.1128/iai.00281-17.

29. Siadat S, Tabaraie B, Behzadiannejad Q, Norouziuan D, Ahmadi H, Najar-Peerayeh S. Bactericidal Activity of Outer Membrane Vesicle of Neisseria meningitidis Serogroup B as a Vaccine Candidate in Animal Model. Iran J Infect Dis Trop Med. 2007;12(36):11-7.

30. Verhoeven D, Perry S, Pichichero ME. Contributions to protection from Streptococcus pneumoniae infection using the monovalent recombinant protein vaccine candidates PcpA, PhtD, and PlyD1 in an infant murine model during challenge. Clin Vaccine Immunol. 2014;21(8):1037-45. doi:10.1128/cvi.00052 
